\title{
Mineração
}

\section{Benefício do adensamento amostral com perfilagem geofísica no planejamento de lavra a curto prazo}

\section{(Benefit of additional samples derived from geophysical logging to short term mine planning)}

\author{
Leandro José de Oliveira \\ Fabrício Souza de Souza \\ Eng. Minas, Mestrandos, LPM / DEMIN / UFRGS \\ E-mail: ljdeoliveira@yahoo.com.br \\ panshito2004@yahoo.com.br \\ João Felipe Coimbra Leite Costa \\ Jair Carlos Koppe \\ Professor, Dr., LPM / DEMIN / UFRGS \\ E-mail:jfelipe@ufrgs.br jkoppe@ufrgs.br
}

\section{Resumo}

Sondagens com amostragem são comumente utilizadas e de extrema importância para o desenvolvimento da pesquisa e do planejamento da lavra. No entanto, esse tipo de informação é cara de ser obtida e, freqüentemente, sondagem exploratória é usada em planejamento de curto prazo. Sondagens exploratórias, invariavelmente, são coletadas em uma malha amostral incompatível com a resolução necessária para estimar blocos de curto prazo. Esse estudo analisa os volumes obtidos com krigagem ordinária a partir de estimativas utilizando distintas fontes de informação: i.e. sondagem com testemunho em malha de $140 \times 140 \mathrm{~m}$ e a partir de dados amostrados por perfilagem geofísica em malha $10 \times 10 \mathrm{~m}$. A interpretação da perfilagem permitiu a inferência dos valores de espessuras das camadas. Foi utilizada, para comparação, uma área-piloto e foram perfilados os furos destinados ao desmonte com explosivos nessa área. Houve uma diferença muito significativa entre o volume estimado por krigagem ordinária com as informações pouco densas de espessuras obtidas por sondagem exploratória, se comparado com o volume real (aproximadamente 26\%). Contudo, praticamente, não houve diferença entre o volume real e o volume calculado pelos dados da perfilagem geofísica. Uma malha de amostragem muito espaçada pode levar a erros grosseiros, caso seja utilizada para planejamento de produção em curto prazo, como demonstra o caso estudado. A perfilagem geofísica pode ser empregada, sem interferir nas operações de lavra, com resultados positivos, de fácil e rápida aquisição com custos, substancialmente, baixos.

Palavras-chave: Perfilagem geofísica, sondagem, estimativa, krigagem, planejamento a curto prazo.

\author{
Paulo Salvadoretti \\ Professor, Dr., LPM / DEMIN / UFRGS \\ E-mail:psalvadoretti@ufrgs.br \\ Gustavo Antônio Bastiani \\ Geólogo, Copelmi Mineração \\ E-mail:gustavo.bastiani@terra.com.br \\ José Adolfo Carvalho Júnior \\ Engenheiro de Minas, Copelmi Mineração \\ E-mail:adolfo@copelmi.com.br
}

\begin{abstract}
Core samples obtained by drilling surveys are a current practice in the mining industry and are extremely important to mineral exploration and mine planning. However, this type of information is expensive to be obtained and is frequently used for short term mine planning. Diamond drill holes are commonly sampled at a large sampling spacing incompatible with the resolution required for short term mine planning. This study compares the volume estimated using data from borehole cores drilled in a grid of $140 \times 140 \mathrm{~m}$ with the volume calculated from data obtained by geophysical logging in a grid of $10 \times 10$ meters. The study analysed the volume of a block delimited by few blast holes. There was a very significant difference between the volume calculated from borehole core samples and the actual volume. However, there was no significant difference between the actual volume and the volume calculated from data obtained by geophysical logging. Thus, it can be concluded that a widely-spaced sampling mesh is not enough to foresee short term production for the case study. However, geophysical logging can be used for this purpose without disturbing mining operations; it also gives immediate results, with easy and fast data acquisition at substantially low costs.
\end{abstract}

Keywords: Geophysical logging, borehole cores, estimation, kriging, short term planning. 


\section{Introdução}

Campanhas de amostragem são práticas corriqueiras e de extrema importância para o desenvolvimento da pesquisa e do planejamento da lavra. O custo de uma campanha pode ser elevado dependendo do método utilizado.

Muitas vezes a malha de amostragem é pouco densa para ser adequadamente utilizada para planejamento da produção de curto prazo. Isto, normalmente, ocorre, devido a restrições orçamentárias e ao alto custo de execução da amostragem por sondagem com recuperação de testemunhos. Para um planejamento de curto prazo, podem ocorrer discrepâncias elevadas entre o valor estimado e o valor real usando dados de uma malha amostral muito espaçada.

$\mathrm{O}$ adensamento de uma malha amostral para um planejamento de curto prazo pode ser realizado com métodos alternativos de amostragem. A perfilagem geofísica é um método de amostragem indireta, eficaz após o desenvolvimento de novas tecnologias para medições de grandezas físicas. Com baixo custo de operação e versatilidade de manuseio, a perfilagem geofísica pode ser realizada, concomitantemente, com as operações de lavra, proporcionando reposta imediata do parâmetro medido, onde a interpretação pode ser realizada desde que seja conhecida a relação entre o parâmetro medido e a litologia.

Os primeiros trabalhos de perfilagem geofísica em carvão foram realizados por Conrad Schlumberger na França em agosto de 1928 . O parâmetro medido era a resistividade, que é similar aos registros de resistividade realizados com os equipamentos de hoje. Por volta do ano de 1965, houve o crescimento do uso de equipamentos de perfilagem geofísica, sendo que os geofísicos da época utilizavam somente o parâmetro de resistividade, normalmente empregado para investigação de água subterrânea. $\mathrm{Na}$ mesma época, foi descoberto que, para algumas áreas, as camadas de carvão podiam ser identificadas devido sua alta resistividade, no entanto, em outras áreas, o arenito também apresenta alta resistividade, dificultando a identificação do carvão. Essa semelhança de resistividade entre o carvão e o arenito também pode ser identificada nos carvões brasileiros, principalmente nos carvões do Rio Grande do Sul.

Esse problema pode ser resolvido com a utilização de sonda para medição da emissão natural de raios gama da litologia, pois essa grandeza se apresenta de forma inversa nessas litologias. Com isso, torna-se possível a identificação da camada de carvão com os registros de resistividade e radiação natural de raios gama (Figura 1).

Sondas de densidade foram utilizadas para exploração de carvão, no final dos anos 60, sendo, inicialmente, empregadas na indústria de petróleo em 1954. Pelo fato de a densidade do carvão ser menor, quando comparada com as litologias no seu entorno, torna-se fácil a sua identificação. Porém a utilização desse tipo de sonda requer o uso de uma fonte radioativa (Hoffman et al., 1982).

Devido ao grande conhecimento da estratigrafia da área utilizada para o estudo de caso, foram utilizados somente os parâmetros de resistividade e radiação natural de raios gama para a identificação da camada de carvão. Esses parâmetros devem apresentar valores altos e baixos, respectivamente, nos carvões do Estado do Rio Grande do Sul. Deve ser lembrado que sempre é observado o contraste. O carvão é identificado devido ao contraste existente dele com as litologias ao seu entorno. Esse tipo de resposta é típica em carvões (Hearst \& Nelson, 1985).

Com base na potencialidade do uso de perfilagem geofísica, na identificação de litologias, desenvolveu-se um estudo para avaliar o benefício do uso da informação de perfilagem no planejamento de curto prazo. O trabalho consiste em comparar o volume de minério calculado somente com dados amostrados por sondagem com testemunho em uma malha de sondagem exploratória (pouco densa) com o volume calculado a partir de dados amostrados por perfilagem geofísica em uma malha amostral densa em uma mesma área. Os volumes obtidos a partir desses dois tipos de dados, com espaçamento amostral distinto, serão comparados com o volume real obtido durante a lavra.

\section{Estudo de caso}

Para desenvolver o trabalho proposto, foi realizado um estudo de caso em um depósito de carvão, denominado Mina do Faxinal. O empreendimento pertence a Copelmi Mineração e está localizado no município de Arroio dos Ratos a aproximadamente $60 \mathrm{~km}$ de Porto Alegre no Estado do Rio Grande do Sul.

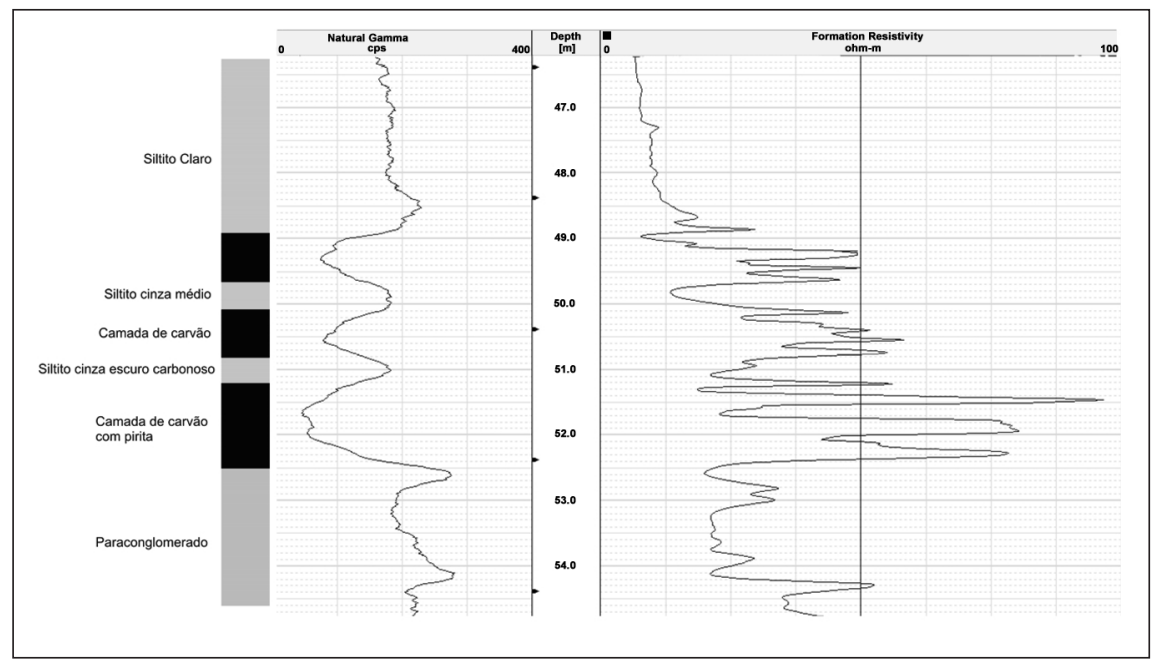

Figura 1 - Exemplo de um registro de perfilagem geofísica dos parâmetros resistividade e radiação natural de raios gama. 
O trabalho iniciou com uma campanha de amostragem, resultando no primeiro banco de dados formado com informações de perfilagem geofísica (denominado de banco de dados A), onde os parâmetros medidos foram a emissão de radiação gama natural e a resistividade da rocha. A partir desses dois parâmetros, foi determinada a espessura da camada de carvão (Figura 1) em cada ponto amostrado. Nessa campanha, foram levantados 36 perfis em malha regular de 10 x 10 metros.

O segundo banco de dados foi construído a partir da medida da espessura das camadas nos testemunhos de sondagem (denominado de banco de dados B) com uma malha amostral média de $140 \times 140$ metros.

Nota-se, pela Figura 2, que existem lacunas que descaracterizam a malha da perfilagem geofísica como regular. Isto ocorreu devido à impossibilidade da identificação da camada através da perfilagem geofísica em alguns pontos, devido ao furo ter sido interrompido antes que toda a camada tivesse sido perfilada.

A partir dos dois bancos de dados, foram calculados os volumes previstos com as diferentes informações disponíveis e comparados com o volume real que foi obtido durante a lavra. A lavra foi, rigorosamente, supervisionada para haver credibilidade na informação que será considerada como a quantidade verdadeira de minério. Para o cálculo dos volumes, foi determinada uma mesma área para os bancos A, B e o real, obtido durante a lavra.

O banco de dados A não sofreu nenhum procedimento geoestatístico, pois a malha amostral é suficientemente densa (informação exaustiva) para permitir o cálculo do volume sem nenhuma interpolação. Foi utilizado o software Surfer $^{\circledR}$ (Golden Software, Inc.) como ferramenta para o cálculo do volume.

O volume obtido na área de estudo com os dados de perfilagem geofísica foi de 1009 metros cúbicos.
Para o banco de dados B, o espaçamento amostral é muito grande, quando comparado ao banco A, e deve haver interpolação em um grid mais denso utilizando krigagem ordinária (Matheron, 1963). O tamanho de bloco a ser estimado coincide com o tamanho da área estudada 40 x $40 \mathrm{~m}$ (aproximadamente $1 / 4$ do espaçamento amostral da campanha de sondagem B).

Para que o valor do bloco estimado apresentasse a mesma orientação da área de estudo, os dados foram rotacionados a fim de que a área obtivesse orientação Norte-Sul (mesma orientação das estimativas), como mostra a Figura 3. Com isto, a área de estudo fica, completamente, definida dentro de um único bloco estimado.

Devido à regularidade da malha amostral, a média desagrupada não apresentou diferença significativa com relação à média dos dados originais. Após, foram calculados os variogramas experi-

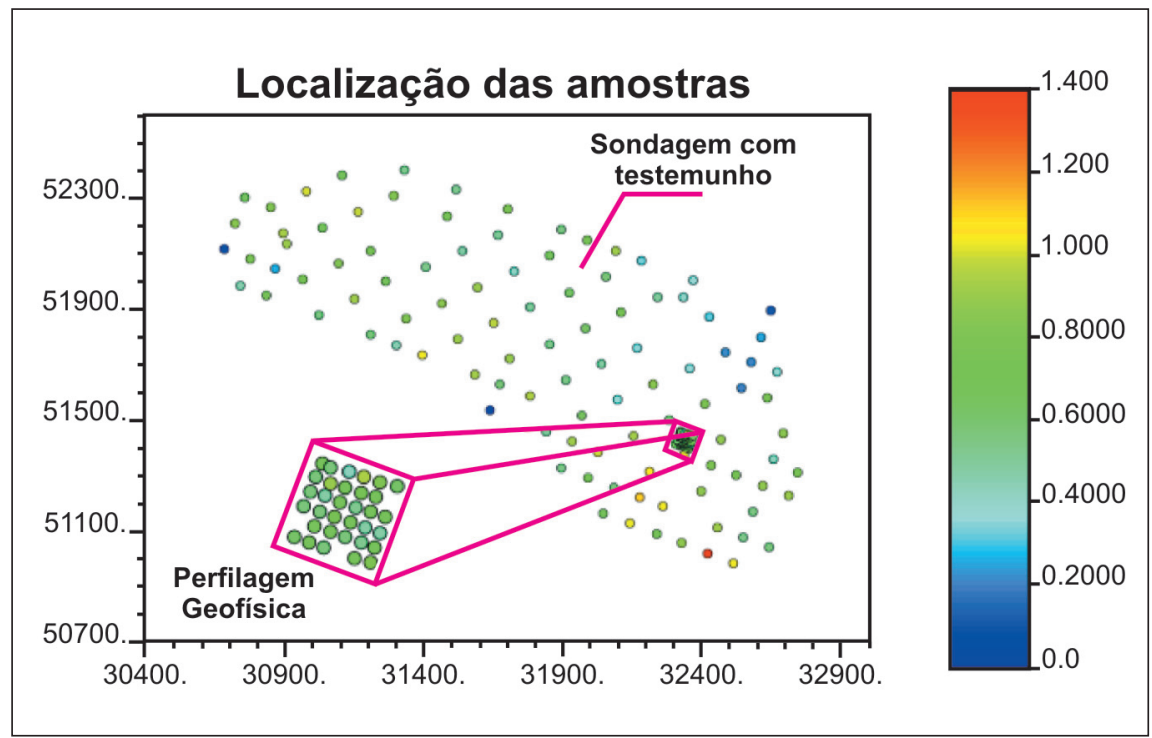

Figura 2 - Mapa de localização da amostras, em destaque o banco A gerado por perfilagem geofísica; as demais amostras pertencem ao banco $B$.

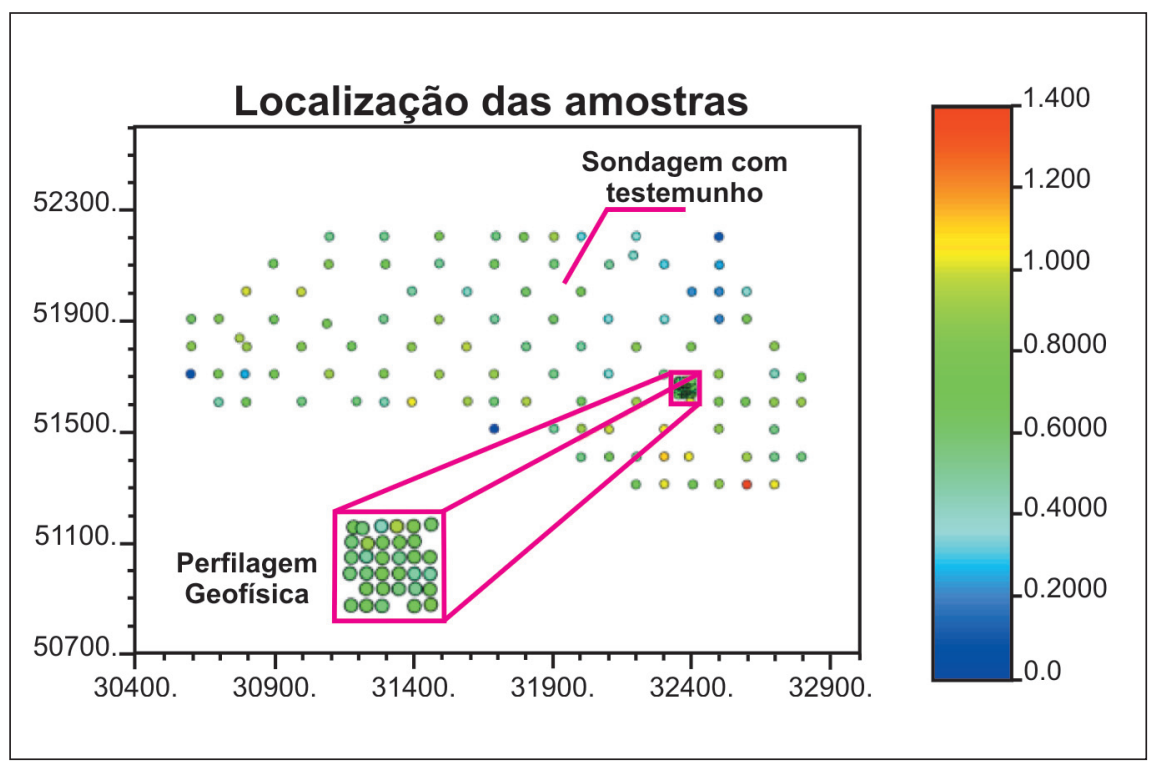

Figura 3 - Mapa de localização das amostras rotacionado. 


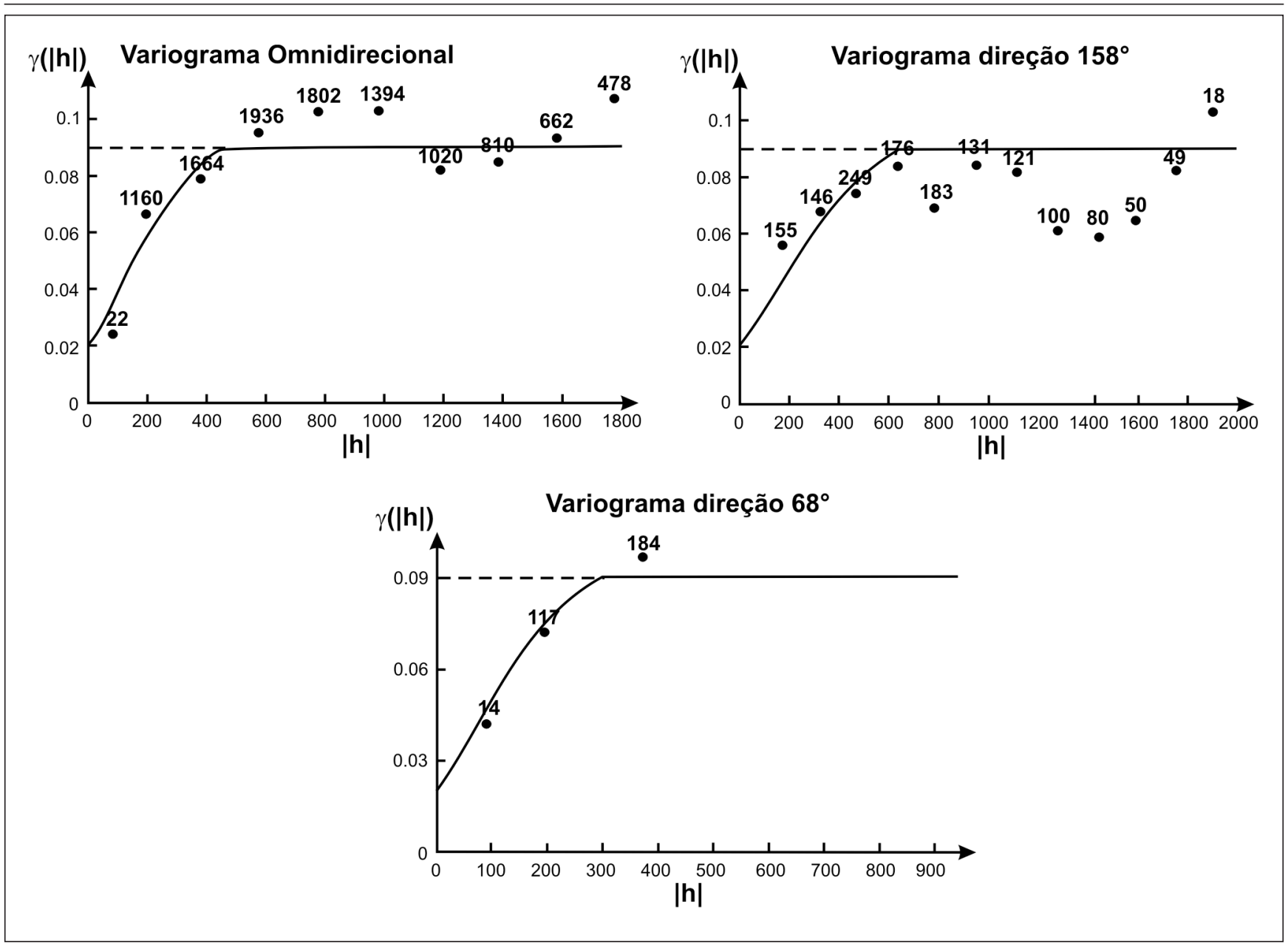

Figura 4 - Variogramas referentes ao banco B.

mentais e tais variogramas foram apresentados junto com seus modelos nas direções $158^{\circ}$ e $68^{\circ}$, de maior e de menor continuidade, respectivamente, considerando o ângulo de rotação do grid.

Os variogramas foram ajustados pelo modelo esférico conforme apresentado na Figura 4.

Os parâmetros do ajuste e as direções principais são apresentados na equação (1) apresentada a seguir.

$\gamma(h)=0.019+0.0715 \operatorname{Sph}\left(\frac{h_{N 68}}{329}, \frac{h_{N 158}}{720}\right)$

onde a equação 1 pode ser traduzida como:

$\gamma(h)=C_{0}+C_{1} \operatorname{Sph}\left(\frac{h_{N 68}}{R_{1}}, \frac{h_{N 158}}{R_{2}}\right)$

$C_{0} \rightarrow$ contribuição do efeito pepita.

$C_{1} \rightarrow$ contribuição da primeira pepita.

Sph $\rightarrow$ ajuste esférico.

$R_{1} \rightarrow$ alcance na direção N68 .

$R_{2} \rightarrow$ alcance na direção $\mathrm{N} 158^{\circ}$.
O método de interpolação empregado foi a krigagem ordinária. Nesse tipo de interpolação, o desconhecimento da média local pode ser filtrado induzindo a soma dos pesos de krigagem igual a 1 (Matheron, 1963).

$$
Z_{O K}^{\bullet}(u)=\sum_{\alpha=1}^{n(u)} \lambda_{\alpha}^{O K}(u) Z\left(u_{\alpha}\right)
$$

$\operatorname{com} \sum_{\alpha=1}^{n(u)} \lambda_{\alpha}^{O K}(u)=1$

onde:

$Z_{O K}^{\bullet}(u) \rightarrow$ valor estinado na posição $u$.

$\lambda_{\alpha}^{O K}(u) \rightarrow$ peso de cada amostra em relação a $u$.

$Z\left(u_{\alpha}\right) \rightarrow$ valor de cada amostra.

$n(u) \rightarrow$ número de amostras utilizadas. 
O volume estimado por krigagem foi de, aproximadamente, $1.271 \mathrm{~m}^{3}$ de carvão da camada $\mathrm{S} 1$, na área de estudo. Esse volume foi obtido pela espessura média estimada por krigagem (média de um bloco) multiplicada pela área estudada.

$\mathrm{O}$ volume verdadeiro foi obtido na lavra do minério. Durante a lavra, foram pesados todos os caminhões envolvidos no transporte do minério. Assim, no final das operações de lavra, foi obtida a tonelagem total do minério "in situ" Considerando a densidade obtida das análises dos testemunhos de $1,8 \mathrm{~g} / \mathrm{cm}^{3}$, torna-se possível determinar o volume.

tonelagem lavrada $=1813 \mathrm{t}$

Volume $=\frac{1813}{1,8}=1007 \mathrm{~m}^{3}$

\section{Discussão dos resultados}

Houve uma diferença muito significativa entre o volume calculado a partir do banco de dados B e o volume real, no entanto, pode-se dizer que não houve diferença entre o volume real e o volume calculado a partir do banco de dados A (Tabela 1).

A Tabela 1 mostra a diferença desprezível entre o volume obtido com os dados do banco A e o volume verdadei- ro. No entanto, a diferença entre o volume obtido com os dados do banco B com o verdadeiro é aproximadamente $26 \%$.

\section{Conclusões}

Ficou claro, nesse estudo, que uma campanha de sondagem com recuperação de testemunho, por ter uma malha amostral relativamente espaçada, não é indicada como cálculo confiável de volume de material contido numa determinada área, para fins de controle de produção a curto prazo.

A perfilagem geofísica, utilizando os parâmetros de resistividade juntamente com radiação natural de raios gama, permite uma boa identificação dos contatos litológicos. Sendo assim, por possuir a facilidade de utilizar os próprios furos destinados ao desmonte, e com uma malha amostral bem densa, a perfilagem geofísica auxilia, de maneira rápida, o cálculo do volume de material contido na área em questão.

Mesmo se tratando de um depósito sedimentar, onde a espessura de cada camada tende a variar muito pouco, o espaçamento da malha amostral influencia, significativamente, na confiabilidade dos cálculos de volume. Como a sondagem por testemunhos é impraticável numa malha tão densa como a malha de furação para o desmonte, devido ao tempo e custo despendido, a perfilagem geofísica mostra-se como a melhor opção
Tabela 1 - Quadro comparativo dos volumes obtidos.

\begin{tabular}{c|c}
\hline Informação & Volume $\left(\mathbf{m}^{\mathbf{3}}\right)$ \\
\hline Banco A & 1009 \\
\hline Banco B & 1271 \\
\hline Verdadeiro & 1007 \\
\hline
\end{tabular}

no processo de controle de produção num curto espaço de tempo, gerando resultados muito próximos da realidade.

\section{Referências bibliográficas}

MATHERON, G . Principles of geostatistic. economic geology. 1963.

HOFFMAN G.L., JORDAN G.R., WALLIS G.R. Geophysical borehole logging handBook for coal explotation. Edmonton, Alberta Canada: The Coal Mining Research Centre, 1982. 270p.

GOOVAERTS, P. Geostatistics for natural resources evaluation. Oxford University Press, 1997.

HEARST JOSEPH, R., NELSON PHILIP, $\mathrm{H}$. Well logging for physical properties. New York: McGraw-Hill Publications, 1985. $571 \mathrm{p}$.

Artigo recebido em 04/05/2007 e aprovado em 01/03/2008. 